\title{
Questes
}

Revue pluridisciplinaire d'études médiévales

Journée d'étude 1 - Trier, classer, organiser | 2022 Trier, classer, organiser

\section{Bête eschatologique, bête sauvage : quelle place pour la bestia dans les ensembles eschatologiques?}

\section{Élise Haddad}

\section{(2) OpenEdition}

Édition électronique

URL : https://journals.openedition.org/questes/5954

DOI : 10.4000/questes.5954

ISSN : 2109-9472

Éditeur

Les Amis de Questes

Édition imprimée

Pagination : $53-68$

ISSN : 2102-7188

\section{Référence électronique}

Élise Haddad, «Bête eschatologique, bête sauvage : quelle place pour la bestia dans les ensembles eschatologiques? », Questes [En ligne], Journée d'étude 1 - Trier, classer, organiser | 2022, mis en ligne le 31 janvier 2022, consulté le 22 février 2022. URL : http://journals.openedition.org/questes/5954 ; DOI : https://doi.org/10.4000/questes.5954

\section{(C) Association des amis de «Questes »}




\title{
Bête eschatologique, bête sauvage : quelle place pour la bestia dans les ensembles eschatologiques?
}

\author{
Élise HADDAD \\ Gahom/AhloMA, CRH-EHESS, Paris/IISER-Pune, Inde
}

Les bêtes sauvages, supposées réelles ou fantaisistes, leurs gueules dentues, menaçantes, grandes ouvertes, sont omniprésentes aux portails et chapiteaux des édifices romans. Mais les différentes formes de lions stylophores sont aussi très répandues, encadrant par exemple les portails de l'Italie du Nord - encore qu'il n'y ait pas, à cet égard, d'exclusivité régionale.

C'est justement dans l'observation de lions à un portail roman que le présent questionnement trouve sa source. Le portail de Beaulieu-surDordogne présente à cet égard une particularité remarquable, qui 1'apparente à quelques édifices de la région (fig. 2) : Moissac, Souillac, pour citer les plus connus ${ }^{1}$. Ces édifices, datés des années 1120-1130, sont ornés de lions aux corps entrecroisés avec virtuosité, formant le corps même du trumeau et soutenant les célèbres tympans monumentaux. À Beaulieu-sur-Dordogne en particulier, le rôle des lions semble osciller

\footnotetext{
${ }^{1}$ Les travaux des années 1970 et 1990 sont encore communément cités pour ce qui concerne l'iconographie du portail de Beaulieu-sur-Dordogne. Bien que le présent article se base sur une interprétation iconographique renouvelée, nous les mentionnons ici. Yves Christe, «Le Portail de Beaulieu, étude iconographique et stylistique», Bulletin archéologique du comité des travaux historiques et scientifiques, nouvelle série, 6, 1971, p. 57-76, et Peter Klein, «Le tympan de Beaulieu: Jugement dernier ou Seconde Parousie?», Cahiers de Saint-Michel de Cuxa, 19, 1988, p. 129-134.
} 
entre deux pôles opposés : positif ou négatif, menaçant ou adjuvant de l'homme ? On ne sait comment les caractériser.

L'examen d'une autre image, une arche de Noé tirée du Beatus de Rylands (Manchester, John Rylands University Library, ms. Lat. 8, daté du dernier quart du XII ${ }^{\mathrm{e}}$ siècle), qui fait partie des manuscrits contenant le commentaire de l'Apocalypse par Beatus, a transformé cette question de caractérisation en un problème de catégorisation: que faire du lion, représentant de la bestia, et de son rôle ambivalent en contexte eschatologique $^{2}$ ? Comment donner une place à cette créature dans le grand ordre des choses? Quelle est sa position dans l'ordonnancement du monde ? Or cette image, tirée du commentaire apocalyptique par Beatus, possède une affinité thématique avec les œuvres sculptées de Beaulieu, tout en appartenant à une tradition iconographique spécifique: les manuscrits du Beatus, sans nécessairement constituer des sources pour la sculpture, témoignent en revanche d'une même lignée d'élaboration graphique autour de l'Apocalypse. De nombreuses similitudes et références en témoignent.

Nous commencerons donc par observer l'image manuscrite, à la fois eschatologique et classificatoire, en analysant la place qu'elle donne à la bête en général, c'est-à-dire d'une part à la bête eschatologique et d'autre part au lion, dans un destin apparemment commun. Puis nous questionnerons, suivant en cela l'indication de notre image classificatoire, la place de la Bestia eschatologique au tympan de Beaulieu-surDordogne, et reviendrons finalement, en bouclant ainsi le cycle, aux lions du notre portail.

\footnotetext{
${ }^{2}$ Pour des considérations générales sur ce manuscrit et des reproductions des images, on se réfèrera à John Williams, The illustrated Beatus: a corpus of the illustrations of the commentary on the Apocalypse, Vol 1-5, London, Miller, 1994-2003.
} 


\section{Que fait la bestia dans une arche de noé ?}

L'image d'arche de Noé (fig. 1) qui nous intéresse est elle-même issue d'un contexte non pas vétéro-testamentaire, mais eschatologique. Elle se trouve en effet au début du Beatus dit de Rylands, au f. 15r. Les manuscrits du commentaire de l'Apocalypse par Beatus s'ouvrent sur des séries de pleines pages enluminées, précédant le texte du commentaire de l'Apocalypse. La succession et le nombre de ces images sont variables selon les manuscrits. Dans le cas du Beatus de Rylands, l'ordre est le suivant :

- une page ornée, mais sans figures ni inscriptions ;

- deux théophanies successives ;

- huit pages représentant les évangélistes ;

- un diagramme généalogique de l'histoire sainte ;

- le combat d'un oiseau et d'un reptile ;

- plusieurs paires de personnages en discussion ;

- enfin, en quinzième page, l'arche de Noé.

En revanche, le Beatus de Saint-Sever, daté de 1060-1070, s'il s'ouvre sur une série d'images très proche de celles du Beatus de Rylands, n'inclut pas d'arche de Noé. D'autres manuscrits du Beatus comportent des images de l'arche de Noé, mais pas nécessairement dans la série de pleines pages qui ouvrent le manuscrit. Ce n'est pas le cas par exemple dans les Beatus de Morgan, d'Urgell, de Facundus, de Gérone, etc. Les images d'ouverture des manuscrits du Beatus ont certes une valeur historique, en ce qu'elles retracent de manière diagrammatique la chronologie ecclésiale et humaine ; elles ont aussi une valeur cosmique : elles mettent en scène des figures théologiques, ou des combats métaphoriques, dont la signification vaut pour le monde entier. 
Le contexte particulier de notre image permet donc de mieux définir son statut. Il ne s'agit pas d'une arche de Noé en tant que simple épisode narratif, au sein d'une succession vétéro-testamentaire. Elle est donnée à voir en préambule au commentaire du Beatus comme l'une des représentations successives fondatrices de l'ordre du monde. C'est en cela qu'il est particulièrement pertinent d'examiner la place attribuée à la bête apocalyptique dans cette composition; plus précisément, cette place semble être révélatrice d'une position au sein d'un classement général.

L'aspect narratif de cette image est de fait assez réduit, et relégué à l'espace périphérique, presque marginal, en tout cas extérieur aux limites de l'arche. Deux oiseaux en sont les personnages agissants. Il y a d'abord la colombe blanche, visible à l'extérieur de l'arche, coupant un rameau ; un second avatar du même oiseau, au sein de la même page, ré-intègre l'arche par la petite section ouvrante du toit. Ainsi, le couple de colombes de l'arche est destiné à être réuni au sein du grand tableau animal déployé sur la page. Le corbeau, en revanche, arrache les yeux des trois cadavres humains disposés en bas de page; il ne retourne pas dans l'arche; le couple des corbeaux est donc divisé et leur case destinée à demeurer dans l'incomplétude.

Mais c'est la valeur classificatrice de l'image qui prend le dessus. Cette priorité est marquée par plusieurs moyens esthétiques : la structure tabulaire, par espèces réparties en couples dans des cases séparées, se différencie de la tradition de l'arche de Noé dans les images antérieures des Beatus. On pourra comparer par exemple l'arche du Beatus de Rylands à celle du Beatus de Gérone, au f. 103r, dans laquelle les animaux ne sont pas séparés dans des cases individuelles, mais interagissent les uns avec les autres ${ }^{3}$.

\footnotetext{
${ }^{3}$ Cathédrale Sainte-Marie de Gérone, Ms. 7.
} 
La mise en ordre va beaucoup plus loin dans l'image du Beatus de Rylands. Les cases ont la même dimension et ils sont répétés côte à côte en une longue série. Leur disposition en six lignes et sept colonnes est en fait complétée par le toit, l'ensemble formant un équilibre visuel entre hauteur et largeur. Plus encore que leur répartition, c'est le grand nombre de cases qui démarque cette image d'autres représentations comparables : six lignes et sept colonnes, soit 42 cases, sans compter les humains - on comparera, par exemple, aux 13 cases (trois lignes de quatre à cinq images) de l'arche de Noé de Saint-Savin ${ }^{4}$. Enfin, dans notre image, la répartition régulière des couleurs formant des diagonales, vient souligner cette valeur d'ordonnancement régulier.

Au sein de ce schéma général, un classement hiérarchique est mis en place, dans lequel prend place la bête eschatologique. Parmi les humains, une séparation entre hommes et femmes est complétée par un tri en fonction de la taille, probablement représentative de l'âge, à en juger par la figure imberbe de l'homme le plus à droite et le plus petit. L'ensemble est centré autour de l'homme de plus haute stature, mais la plus grande des femmes empiète sur l'espace de son corps et de son vêtement dans des proportions notables, se désignant ainsi comme à la fois subordonnée à l'homme principal, Noé, mais néanmoins son homologue dans une hiérarchie des femmes analogue à la hiérarchie des hommes.

Aux étages des multiples espèces non-humaines, un premier tri évident sépare les trois rangées supérieures, dédiées aux créatures ailées, des trois lignes inférieures, où s'ordonnent les créatures terrestres. Au sein de ces deux grandes catégories s'établissent des subdivisions : parmi les oiseaux, la première ligne accueille les oiseaux appartenant à la sphère

\footnotetext{
${ }^{4}$ Les célèbres peintures murales sont datées du début du XII ${ }^{\mathrm{e}}$ siècle ; l'Arche de Noé se trouve parmi les épisodes représentés sur la voute de la nef.
} 
domestique, des oiseaux utiles à l'homme. Outre la colombe et le corbeau, dont la collaboration avec l'homme est spécifique à cette scène, on y trouve le coq et la poule, ainsi qu'un couple canne-canard, dans les deux cas caractérisés par des marques fortes d'appartenance à une espèce. Tout à droite, si les marques physiologiques de l'espèce sont moins distinctives, c'est la situation, sur un perchoir et les pattes entravées par des liens, qui permettent de désigner la situation du dernier couple, peutêtre des faucons. Deux objets de facture humaine, et notamment le chaudron accroché, marqueur par excellence du foyer, complètent cette première série. La deuxième ligne réunit des animaux non domestiques, mais appartenant à la faune commune dans l'écosystème européen : on reconnaît deux hiboux et peut-être, à la deuxième case en partant de la droite, deux hérons. Enfin, s'il est impossible d'identifier toutes les espèces présentes à la troisième ligne, il est clair que celles qui présentent des caractéristiques très distinctives, et qui sont donc reconnaissables, appartiennent à des espèces exotiques : on repère ainsi les paons et les perroquets. En somme, au sein du monde aviaire, le classement se fait depuis les êtres à proprement parler domestiques, au plus haut et au plus près de l'homme, vers les animaux seulement familiers, puis exotiques.

Sous ces représentants célestes sont disposées, de nouveau sur trois rangs, les espèces terrestres. La polarité domestique/sauvage est de nouveau opérante. Mais la répartition est différente: un rang pour les espèces domestiques, comme l'âne, le bœuf, le cheval, les ovins, les caprins, et deux rangs pour des bêtes moins proches de l'humain, relevant du sauvage familier, comme le cerf, ou de l'exotique, voire du monstrueux. Parmi ces animaux dont le statut, entre lointain et imaginaire, n'est pas absolument clair, tous ceux qui présentent des formes hybrides entre animaux terrestres et ailés sont regroupés au second rang. Ainsi, dans la polarité axiologique qui est à l'œuvre, les 
ailes, quoique ne compensant pas tout à fait le statut de bête lointaine ou mythique, éventuellement même menaçante, apportent tout de même un petit surclassement: elles justifient l'appartenance à une catégorie intermédiaire, au second rang plutôt qu'au troisième.

On s'attend dès lors à voir apparaître à la dernière ligne la lie de la création, le pire de la faune terrestre, voire infra-terrestre. De fait, plusieurs apparitions frappantes s'y succèdent. Tout à gauche de la ligne, deux créatures simiesques semblent faire preuve d'une pudeur toute humaine. Il s'agit probablement de créatures exotiques, dans la tradition plinienne $^{5}$; leur comportement proche de celui des humains apporte un surcroît d'inquiétante étrangeté. À leur suite, deux animaux particulièrement remarquables prennent place: des bêtes à sept têtes. L'image ne cherche en rien à normaliser leur présence, étonnante ici : c'est le seul couple qui occupe deux cases distinctes. Dans le contexte de pages liminaires au commentaire de l'Apocalypse, il est impossible d'ignorer l'allusion aux bêtes à sept têtes qui parsèment le texte et la narration. $\mathrm{Du}$ reste, ce caractère eschatologiqueapocalyptique est renforcé, nous semble-t-il, par la présence en fin de ligne d'un objet architecturé que nous identifions comme une arche d'alliance : son aspect est similaire au motif de l'arche représentée dans les illustrations du Beatus correspondant à la venue de la bête de $1^{\prime}$ Abysse ${ }^{6}$. Le contraste avec la première case du tableau, occupée par un objet du foyer, est

\footnotetext{
${ }^{5}$ Rappelons ici que les Histoires Naturelles de Pline ont alimenté tout l'imaginaire médiéval des créatures vivant en Orient. Parmi elles, les cynocéphales et êtres simiesques sont les plus proches du couple de créatures représentées dans l'arche du Beatus de Rylands. Voir Jacques Le Goff, La civilisation de l'Occident médiéval, Flammarion, Paris, 2008. Pour un accès au texte, on se réfèrera à Pline l'Ancien, Histoire Naturelle, Livre VI, Paris, Les Belles Lettres, Collection des Universités de France, 1980.

${ }^{6}$ Commentaire d'Apocalypse XI, 7. Les images se trouvent, dans le Beatus de Rylands, au f. 141r, mais aussi, de manière plus frappante dans le Beatus de Turin (Biblioteca Nazionale Universitaria di Torino, Ms : I.II 1, ancien Lat.93) au f. 130v et le Beatus de San Millán (Real Academia de la Historia, Cod. 33), f. 158r, etc.
} 
saisissant. Nous passons ici sur le troisième objet de la composition, bien évidemment inclus dans cette dynamique, mais qui ne nous semble pas jouer un rôle essentiel dans l'argumentaire que nous déroulons.

L'introduction de la bête à sept têtes a de quoi surprendre. L'arche de Noé est supposée contenir l'ensemble des animaux créés. La bête apocalyptique, a priori, telle que comprise dans les commentaires, a une valeur métaphorique : elle représente les malheurs à venir au moment de la fin des temps, et les êtres mauvais, porteurs de ces maux — voire, pour les interprétations moins eschatologiques de l'Apocalypse, le mal dans le monde présent - mais certainement pas un animal existant. Quand bien même on tiendrait compte d'une compréhension plus littérale de ce passage, ce qui semble peu avéré dans les documents, alors cette bête n'aurait pas été créée à l'époque ancienne du Déluge. Enfin, dans son unicité, cette bête n'a pas de caractère sexué, ni de vocation à la reproduction par un couple de même espèce. À tout point de vue, son inclusion dans l'ordre de l'arche pose problème. Sa présence rompt avec la logique d'organisation du monde vivant opérée par l'image. Elle dérange et irrite. Elle mène nécessairement à une réflexion sur sa place dans la création, et dans l'ici-bas.

Enfin, mentionnons encore un trait remarquable: les voisins immédiats de ces deux bêtes apocalyptiques sont un couple de lions, bêtes sauvages eux aussi, quoiqu'il soit logique de les catégoriser au sein des créatures. Ce voisinage entre bête apocalyptique et lion n'est pas unique et sa récurrence nous semble remarquable.

C'est précisément cette réflexion sur le classement de la bête que nous voulons poursuivre en partant de l'image du Beatus de Rylands et en la confrontant à une autre œuvre romane: le portail sculpté de Beaulieu-sur-Dordogne. La mise en problème visuelle de la place des bêtes, et parmi elles celles de la bête eschatologique et du lion, côte à 
côte, n'est en effet pas unique à cette représentation de l'arche. Cette même question permet de mieux comprendre les dynamiques qui traversent l'ensemble sculpté de Beaulieu-sur-Dordogne.

\section{Les bêtes au tympan de Beaulieu-sur Dordogne}

Le tympan de Beaulieu-sur-Dordogne (fig. 2) est une des œuvres majeures de l'art roman monumental. Nous avons montré ailleurs qu'il ne représente pas un Jugement Dernier, mais d'autres figures et évènements apocalyptiques. Deux types de bêtes apocalyptiques s'y donnent à voir, respectivement au registre inférieur et médian du tympan ${ }^{7}$.

$\mathrm{Au}$ registre inférieur d'abord, trois bêtes sont présentées, sur fond de rosaces végétales : selon nos conclusions ${ }^{8}$, la bête de la terre, la bête de la mer et le dragon de l'Apocalypse' ${ }^{9}$. La présence de la bête de la mer, deuxième en partant de la gauche, est à cet égard la plus intéressante pour une comparaison avec l'image du Beatus de Rylands. Il s'agit là aussi d'une bête à sept têtes, quoique l'option graphique choisie pour accumuler ces têtes soit tout à fait différente. Notons au passage que cette bête à sept têtes porte également sept mamelles, dans une évocation sexuée assez rare - peut-être développement visuel d'une association à des figures féminines, comme celle de la grande prostituée, ou peut-être forme de réalisme et de carnalité rapprochant cette bête des animaux terrestres dotés d'une existence corporelle.

Au registre intermédiaire, néanmoins, d'autres bêtes nous semblent plus intéressantes encore. Quoiqu'elles ne correspondent pas littéralement

\footnotetext{
${ }^{7}$ Élise Haddad, «Common space or cleft places? The example of Beaulieu, an architectural and figured space», dans Place and Space in the Medieval World, dir. Meg Boulton, Jane Hawkes, Heidi Stoner, New York/Abindgon, Routledge/Taylor and Francis, 2018, p. 114-125.

${ }^{8}$ Voir ibid., p. 116.

${ }^{9}$ Apocalypse, XII, 3 et XIII, 11.
} 
à la même figure que celle du Beatus de Rylands, elles font fonctionner la question de la bestia et de sa catégorisation avec plus de puissance. Il s'agit de quatre locustes ${ }^{10}$, créatures envoyées par Dieu sur la Terre pour $\mathrm{y}$ torturer les hommes ${ }^{11}$. Elles sont représentées au tympan de Beaulieu par des créatures léonineshybridées à la fois par des caractères empruntés à l'aigle, et d'éléments végétaux.

Ces deux registres correspondent donc, là encore dans une logique qui s'apparente au classement catégoriel, à deux types de bêtes apocalyptiques. Les unes, au registre inférieur, sont des représentations univoques des forces du mal. Les autres, au registre médian, sont des créatures complexes, provoquant la souffrance mais néanmoins envoyées par Dieu.

L'opposition structurelle entre ces deux registres parallèles, respectivement inférieur et intermédiaire, est d'abord marquée par plusieurs éléments de séparation. D'abord, la délimitation est redoublée. Elle est marquée d'une part par la corniche et d'autre part par la ligne de double nuée. Elle est donc bien plus hermétique que la séparation simple entre registre médian et demi-disque supérieur, d'autant plus que cette dernière est presque totalement traversée, en tout cas largement amenuisée sous les pieds du Christ. On notera également que la corolle végétale qui ponctue la première voussure vient s'insérer et s'épanouir exactement à la limite entre les deux registres, les distinguant ainsi l'un de l'autre.

Mais surtout, cette différenciation se manifeste dans un certain nombre de traits visibles dans l'iconographie. À la nature ailée des locustes s'oppose ainsi l'absence d'ailes des bêtes au registre inférieur caractère qui ne peut, évidemment, être justifié par une simple référence

\footnotetext{
${ }^{10}$ Voir Élise Haddad, «Common space or cleft places ? », art. cit., p. 118.

${ }^{11}$ Apocalypse, IX, 3.
} 
au texte, puisque, d'une part, l'aspect des créatures s'en écarte à bien des égards, et s'inscrit plutôt dans la tradition visuelle que dans une mise en image du verbe et que, d'autre part, on le sait, le dragon peut être représenté muni de deux ailes, comme c'est le cas dans un certain nombre d'images, de l'Apocalypse de Trèves à des manuscrits tardo-médiévaux, et notamment, au sein de la même tradition dans le Beatus de San Millán ${ }^{12}$. Or ce choix n'a pas été fait à Beaulieu. Les ailes, ainsi que d'autres éléments physiologiques empruntés à l'aigle, et aux nuées sur lesquelles courent les locustes, les associent au monde céleste.

\section{Les lions}

Revenons aux lions, point de départ de notre réflexion. Tournonsnous vers la base du trumeau, soutenant le tympan que nous venons de quitter. Elle est très abîmée aujourd'hui, mais une observation attentive sur place, rendue ici par une proposition dessinée (fig. 3), permet de rendre compte de ce qu'elle représentait. La vue de trois-quarts gauche, en première ligne de la figure 3 , est celle qui permet d'observer avec le plus de facilité l'enchevêtrement des trois corps léonins. Deux lions se croisent, en diagonales. Leurs arrière-trains s'appuient, respectivement, contre la colonnette de gauche pour celui qui est au premier plan, et contre la colonnette de droite pour le suivant. Leurs poitrails plongent alors vers les angles inférieurs du trumeau, et les deux têtes passent sur les faces latérales. Dans la vue de trois-quarts gauche, on distingue la tête du lion, servant de piédestal au personnage dont les pieds ont été abîmés par le temps.

Ici, un premier élément se dégage : cette pyramide acrobatique des corps de lions, servant par ailleurs de socle aux atlantes, est une mise en

\footnotetext{
12 Beatus San Millán, fin du $\mathrm{X}^{\mathrm{e}}$ siècle, Madrid, Real Academia de la Historia Codex 33, f. 165r.
} 
scène forte de leur rôle en tant que porteurs et adjuvants des hommes du trumeau. Ils contribuent, tout en restant en cela subordonnés aux hommes, à porter l'ensemble architectural et l'ensemble sémantique du trumeau et du tympan tout entiers.

Néanmoins, la vue de trois-quarts droite, en deuxième ligne de la figure 3 , nous permet d'observer un deuxième phénomène. Le lion dont le corps forme le premier plan de la face du trumeau possède également une tête, visible du côté droit. Or cette tête, renversée, est dotée de deux solides mâchoires, qui viennent cette fois non pas soutenir, mais bien au contraire prendre en étau la jambe du personnage humain - très abîmé - au bas du trumeau. Que faire de cette démonstration d'agression ? Elle suggère une dangerosité du lion, contrevenant à son rôle positif de soutien à l'architecture. Pourquoi les lions sont-ils représentés dans cette ambivalence entre force nécessaire et socle de l'ensemble, mais aussi menace sérieuse ? Et surtout, en quoi a-t-il été pertinent d'inclure cette représentation dans l'ensemble du portail de Beaulieu?

C'est ici que nos différents exemples prennent sens les uns par rapport aux autres. Les Bêtes apocalyptiques sont nombreuses au tympan de Beaulieu. Certaines semblent absolument négatives, mais ce n'est pas le cas de toutes, et notamment des locustes : envoyées par Dieu pour réaliser son action, mais en produisant souffrance et destruction. Il nous semble que ce parallèle entre locustes torturant deux hommes et lions soutenant et mordant à la fois les atlantes n'est pas une coïncidence, qu'elle est au contraire une manière de mettre en question à la fois le rôle de la bête apocalyptique, et celle de la bête dans la création présente. Elle est du reste rendue plus visible encore par l'analogie de posture entre, d'une part l'atlante à la face du trumeau, juché sur les lions, mais dont le côté droit nous révèle qu'il est aussi potentiellement menacé par leur 
morsure et, d'autre part, l'homme torturé par les locustes du côté droit du registre médian. L'attitude corporelle, au cou cassé vers la droite, ainsi que la physionomie, rendent visible ce rapprochement.

Or il nous semble qu'une clef thématique essentielle de compréhension du portail est la thématique de l'épreuve : ce que les locustes font subir aux hommes, c'est une épreuve nécessaire avant la venue de la délivrance et de la fin des temps ${ }^{13}$. Ce que l'atlante porte, ce à quoi il doit résister, c'est l'épreuve qui lui permet de tenir bon, et de tenir haut l'œuvre du tympan. Cette thématique résonne non seulement dans ces deux motifs, mais dans l'ensemble du portail : épreuve collective, morsure de la tentation infligée à l'individu. C'est à travers ces évènements que l'homme peut cheminer à travers le tympan, en direction du monde céleste. Dès lors, le rôle des locustes et des lions est effectivement analogue, et il est essentiel. Le discours porté par le portail de Beaulieu-sur-Dordogne assigne donc une mission à la bête féroce au sein du plan divin : celle d'infliger une souffrance dotée de sens, la souffrance de l'épreuve.

Ainsi est circonscrite la place de la bestia, bête sauvage ordinaire dans la création, en passant par la métaphore de la Bestia apocalyptique, la Bête néfaste exemplaire. Notre portail sculpté partage en cela une préoccupation du commentaire livresque de l'Apocalypse de Rylands, qui lui est postérieur d'un demi-siècle. Il dialogue avec l'énigme, la question posée par l'image de l'arche, dernière image de préambule avant un commentaire de l'Apocalypse. Il s'agit dans les deux cas de trouver une place à la bestia dans l'ordonnancement du monde créé. Pourquoi un créateur bon et tout puissant a-t-il placé dans le monde des bêtes mauvaises ? Et dans l'image de l'Arche comme au tympan de Beaulieu, c'est par l'analogie avec une bête apocalyptique que cette question peut

\footnotetext{
${ }^{13}$ Voir Élise Haddad, « Common space or cleft places ? », art. cit., p. 118-119.
} 
être posée, et une forme de réponse apportée. À cet égard, une réflexion commune se donne à voir dans les deux œuvres apocalyptiques que sont le manuscrit illustré du commentaire de Beatus et le grand portail de Beaulieu-sur-Dordogne.

Ces observations rendent compte d'une préoccupation grandissante pour le classement au XII ${ }^{\mathrm{e}}$ siècle, et en l'occurrence pour le classement de la bête, de la créature mauvaise au sein d'une création bonne. Elle se manifeste dans la tradition des Beatus par l'élaboration d'une arche de Noé remarquablement complexifiée et ordonnée. Dans le même siècle, mais probablement sans interaction directe avec ce manuscrit, le portail de Beaulieu rend visible un questionnement similaire sur la catégorisation de la Bestia apocalyptique et de la bestia terrestre, à travers la figure du lion.

Dans les deux cas, le questionnement sur la nature des bêtes sauvages est travaillé et résolu par une mise en ordre analogiste, au sens descolien ${ }^{14}$. Les divisions, subdivisions, et analogies catégorielles constitutives de l'arche de Noé renvoient en effet inévitablement à la caractérisation d'une ontologie analogiste chez Philippe Descola, pour qui les redoublements et les subdivisions de catégories sont une caractéristique cruciale des mondes de l'analogie. Quant au passage par le paradigme de la bête apocalyptique pour comprendre le rôle et la place de la bête sauvage sur terre, son mode d'ordonnancement du monde par analogie, en l'occurrence entre l'ordre eschatologique, l'ordre terrestre, et le parcours spirituel, ne fait pas de doute. Ces deux objets, quoique très différents par les moyens graphiques mis en ordre, se recoupent donc non seulement par leur thématique globale et les créatures qu'elles

14 «Analogiste» désigne ici non seulement un fonctionnement analogique, mais l'appartenance à l'une des quatre ontologies telles que définies par Philippe Descola. Voir Philippe Descola, Par-delà nature et culture, Paris, Gallimard, 2005. 
convoquent, l'un permettant de comprendre l'autre, mais aussi par une appartenance commune à la même dynamique d'ordonnancement du monde, et au même type, analogiste, de cette grande mise en ordre.

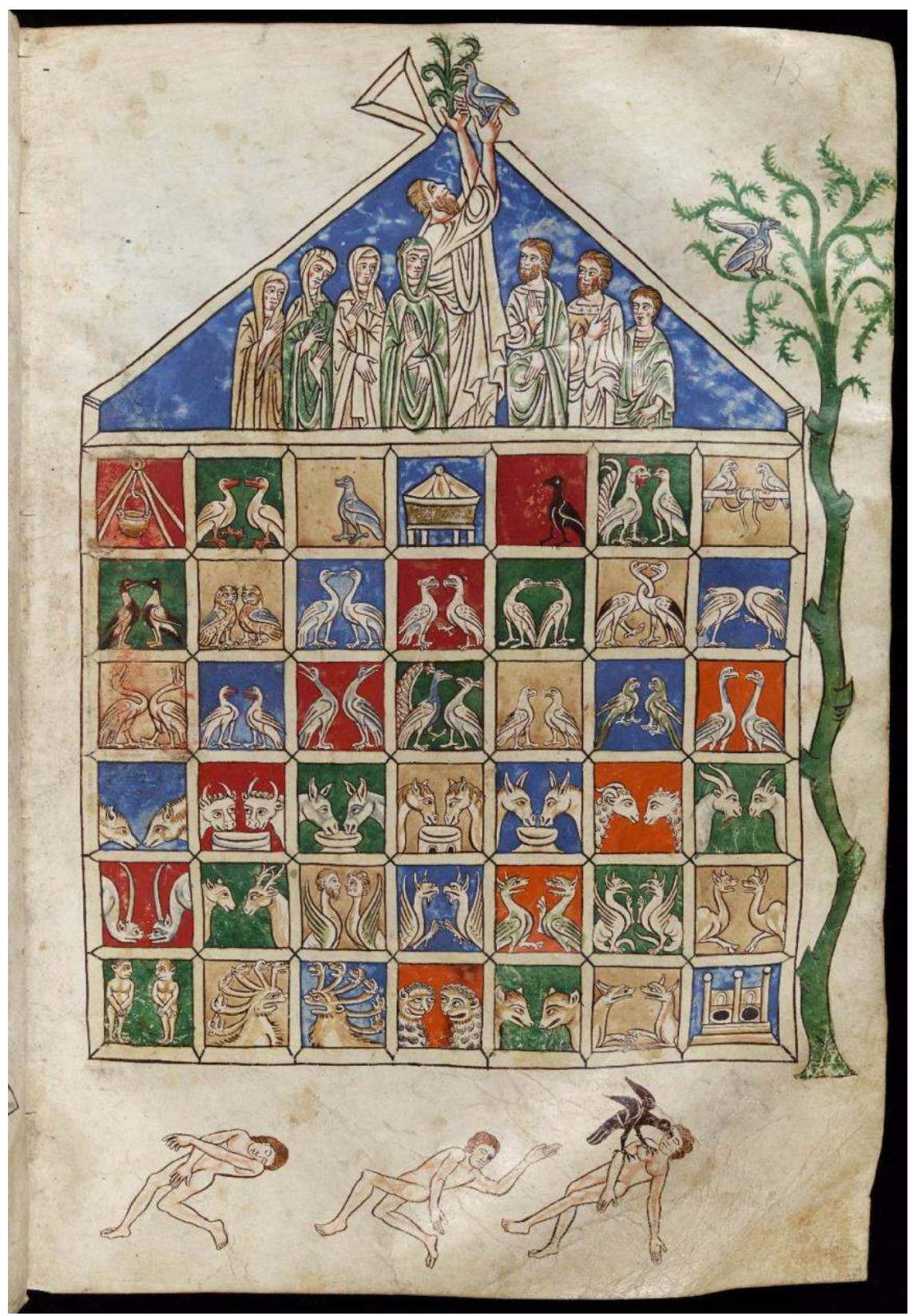

Fig. 1. MANChester, JOHn Rylands Library, Ms LAt. 8, F. 15R. 


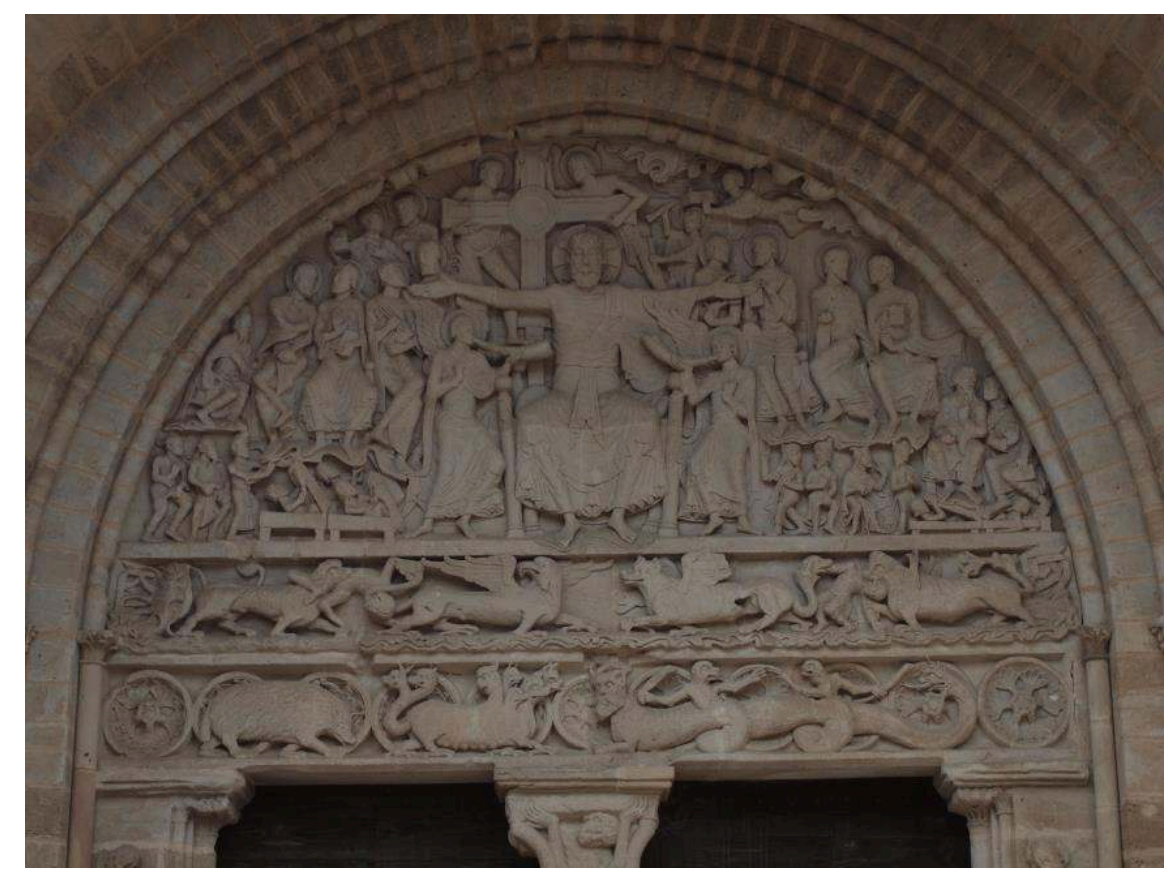

Fig. 2. Abbatiale Saint-Pierre de Beaulieu-sur-Dordogne, Correze, tympan Sud. PHOTOGRAPHIE DE L'AUTEUR.

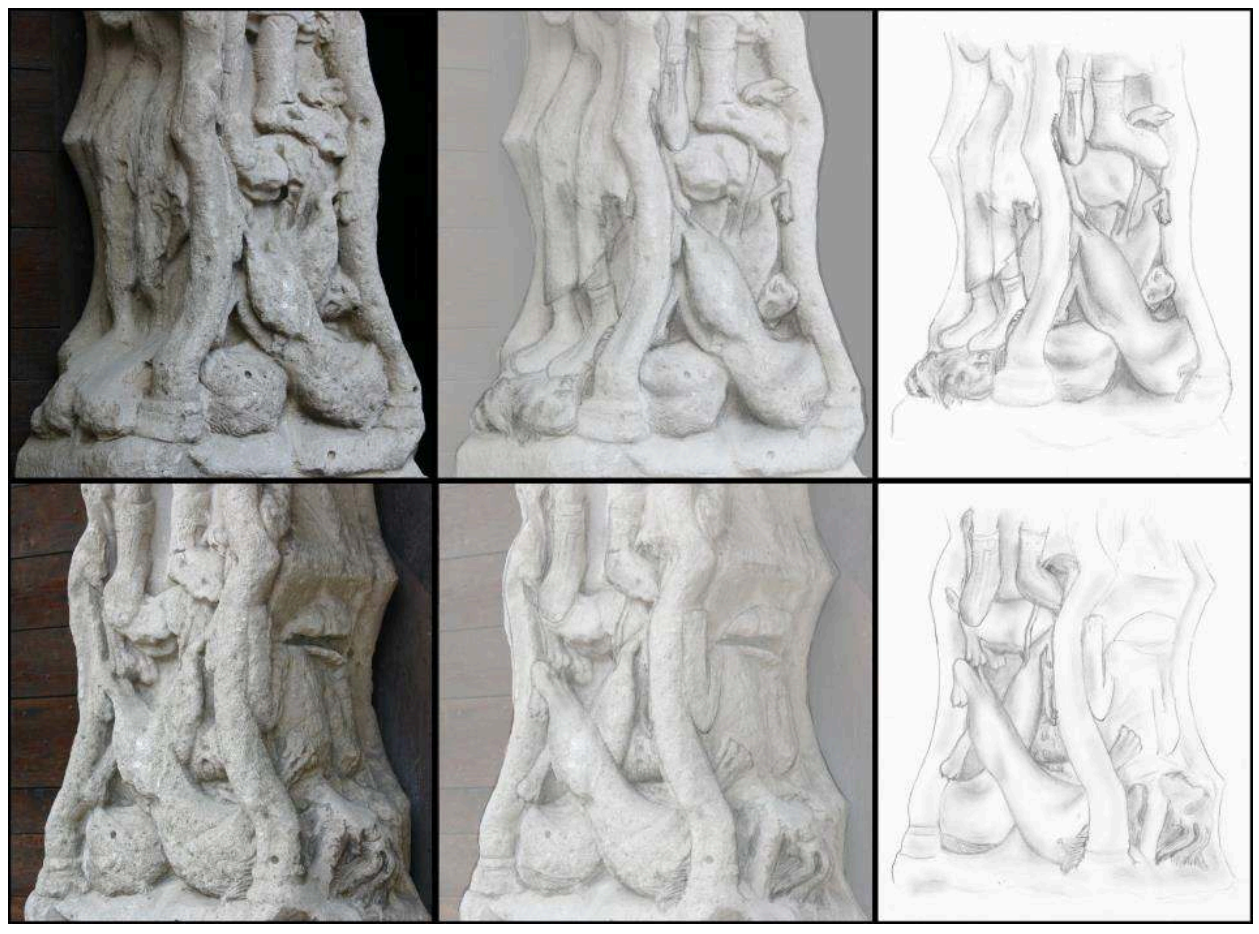

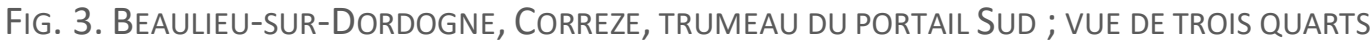
GAUCHE ET DE TROIS QUARTS DROITE ; DANS L'ORDRE : PHOTOGRAPHIE, DESSIN SUPERPOSE A LA PHOTOGRAPHIE, ET DESSIN ORIGINAL ; DESSINS ET PHOTOGRAPHIES DE L'AUTEUR. 